Pathologe 2010 · [Suppl 2] 31:312-313

DOI 10.1007/s00292-010-1299-9

Online publiziert: 22. Juli 2010

๑) Springer-Verlag 2010

\author{
V. Krenn ${ }^{1} \cdot$ K. Hauptmann ${ }^{2}$ \\ ${ }^{1}$ Zentrum für Histologie, Zytologie und Molekulare Diagnostik, Trier \\ ${ }^{2}$ Institut für Pathologie, Charité-Universitätsmedizin Berlin, \\ Charité Campus Mitte (CCM), Berlin
}

\title{
Sitzungsbericht der AG Orthopädische Pathologie
}

\section{Vorsitzende der Sitzung}

- Prof. Dr. med. V. Krenn (Zentrum für Histologie, Zytologie und Molekulare Diagnostik, Trier)

- PD Dr. K. Hauptmann (Institut für Pathologie, Charité-Universitätsmedizin Berlin)

Die seit zwei Jahren bestehende Arbeitsgemeinschaft (AG) für Orthopädische Pathologie traf sich im Rahmen der 94. Jahrestagung der Deutschen Gesellschaft für Pathologie (DGP) in Berlin und war mit sieben freien Vorträgen und fünf Posterbeiträgen vertreten.

Die Abstracts sind im Ergänzungsband der Zeitschrift Der Pathologe publiziert.

Der Focus dieser Arbeitsgemeinschaft liegt in der Förderung und Verbreitung von experimentellen/wissenschaftlichen Daten sowie in der Erarbeitung und Diskussion histopathologischer/diagnostischer Kriterien für neoplastische, entzündliche, degenerative und metabolische Erkrankungen des Bewegungsapparates.

In der diagnostischen Kooperation zwischen Pathologen und Orthopäden/ Unfallchirurgen spielen quantitativ gesehen entzündliche, infektiöse und degenerative Erkrankungen eine größere Rolle als neoplastische Erkrankungen des Knochensystems und des Bewegungsapparates.

Diese Tatsache berücksichtigend, wurden vier Themen aus dem Bereich Infektion, Entzündung, Degeneration und drei Themen aus dem Bereich Neoplasie vorgestellt.

Knösel und Mitarbeiter (Jena) stellten in einer Übersicht den solitären fibrösen Tumor (SFT) vor. Diese Tumorform wur- de 1870 in Leipzig beschrieben und stellt einen mesenchymalen Tumor mit heterogenem Erscheinungsbild und unterschiedlicher Dignität dar. In einer immunhistochemischen Analyse wurden die Marker $\mathrm{CD}_{34}$, BCL2, CD99, glattmuskuläres Aktin, S10o, Panzytokeratin und Ki67 in den Subtypen des SFT (fibröse Variante, zelluläre Variante, fettzellreiche Variante, riesenzellreiche Variante und maligne Variante) analysiert. Im vorliegenden Kollektiv wurden $79 \%$ als fibröse Variante, $17 \%$ als zelluläre Variante, $2 \%$ als fettzellreiche Variante und $1 \%$ als Riesenzellvariante klassifiziert, 3\% wurden als maligne Variante diagnostiziert. In der malignen Variante besteht interessanterweise eine reduzierte CD34-Expression. Die funktionelle Bedeutung der reduzierten CD34-Expression im Hinblick auf das maligne Potenzial ist ungeklärt und Thema weiterer Analysen.

Der Vortrag von Ströbel und Mitarbeitern (Mannheim) beschäftigte sich mit der „MYC-high-level-Genamplifikation“ in so genannten primären und sekundären Angiosarkomen. Sekundäre Angiosarkome sind assoziiert mit einem chronischem Lymphödem oder einem Zustand nach Bestrahlung. In einer Array-CGH-Analyse (28 primäre Angiosarkome, 33 strahleninduzierte Sarkome) wurden molekulare Alterationen analysiert. Die häufigste Alteration war eine „High-level-Amplifikation" am Chromosom 8q24.21 (50\%) gefolgt von einer 1op12.33- (33\%) und einer 5935.3- (11\%) Alteration. Interessanterweise wurde eine „High-level-Amplifikation" von MYC am Chromosom 8q24.21 ausschließlich in den sekundären, strah- leninduzierten Angiosarkomen nachgewiesen. Diese Alteration könnte das Verständnis der Angiosarkomgenese ermöglichen und die Grundlage für eine molekulare Diagnostik darstellen.

Slansky und Mitarbeiter (Dresden) berichteten über die Anwendung der multikategoriellen und binären ROC- („receiver operating characteristic“-) Analyse auf den dreikomponentigen Synovialitisscore nach Krenn [1]. Der Synovialitisscore wurde in 666 Fällen bestimmt: Normalgewebe $(n=33)$, posttraumatische Arthropathie $(n=29)$, Osteoarthrose $(n=221)$, Psoriasisarthritis $(n=42)$ und rheumatoide Arthritis $(n=341)$. Die Diskriminierungsfähigkeit des Synovialitisscores und der einzelnen drei Bewertungskomponenten (synoviale Deckzellschichtverbreiterung, Zellularität des synovialen Stromas und leukozytäre Infiltration) wurden quantifiziert mittels des AUC- („area under the curve“-) Verfahrens der ROC-Analyse und des HUM- („hypervolume under the manifold“-) Verfahrens. Alle drei Komponenten zeigten einen Unterschied zwischen Normalgewebe (AUC 0,87$0,98)$, rheumatoider Arthritis gegen Osteoarthrose und posttraumatischer Arthritis (AUC 0,85). Die AUC-Werte der synovialen Deckzellschichtverbreiterung und der synovialen Zellularität korrelierten $(\mathrm{R}=0,94)$ mit den AUC des gesamten Scores mehr als die Werte der entzündlichen Infiltration $(\mathrm{R}=0,60)$. Die multikategorielle ROC-Analyse ordnete die diskriminatorischen Fähigkeiten des dreikomponentigen Synovialitisscores weit höher ein als die der einzelnen Komponenten. Die HUM-Werte für den kompletten 
Score betrugen 0,62, für die Zellularität des synovialen Stromas o,014, für die Verbreiterung der synovialen Deckzellschicht 0,075 und für die leukozytäre Infiltration o,0005. Hieraus ist abzuleiten, dass die Kombination der drei Merkmale des Synovialitisscores zu einer weit höheren diagnostischen Sicherheit führt als die Verwendung einer der einzelnen Komponenten. Dieser Befund unterstreicht die Notwendigkeit der „Dreikomponentigkeit des Synovialitisscores“.

Herr Otto und Mitarbeiter (Trier) berichteten über die Detektion von minimalen bakteriellen Infektionen in der Synovialmembran und in der periprothetischen Membran von insuffizienten Endoprothesen. Hierzu wurde ein bereits in der Sepsisdiagnostik verwendetes Testverfahren (VYOO, Jena, Deutschland) auf Synovialgewebe, periprothetisches Gewebe und Gewebe der Neosynovialis adaptiert. Mittels dieses Testverfahrens können 40 unterschiedliche Bakterien, Pilze und 5 Resistenztypen detektiert werden. Von 20 Patienten wurde periprothetisches Gewebe untersucht. Sämtliche Infektionen und Resistenzen, die mittels dieses Verfahrens nachgewiesen wurden, konnten in der mikrobiologischen Analyse bestätigt werden. Dieses Testverfahren kann somit bei infektionspathologischen Fragestellungen, insbesondere bei so genannten „Low-grade-Infektionen“ eingesetzt werden.

Die Arbeitsgruppe aus Graz (Österreich) von Herrn Alzoughbi und Mitarbeitern berichtet über die Adipose-Triglyzerid-Lipase- (ATGL-) Expression in Leiomyosarkomen und Leiomyomen. In dieser Analyse (33 Leiomyome und 16 Leiomyosarkome) wurde die immunhistochemische Expression der ATGL analysiert und im Hinblick auf divergente Expressionsprofile bewertet: 93\% der Leiomyome zeigten eine starke granuläre zytoplasmatische Reaktivität, eine schwache Reaktivität war nur in einem Fall nachweisbar. 88\% der Leiomyosarkome waren negativ oder schwach positiv. Myxoide Leiomyosarkome zeigten eine diffuse bis schwache Reaktivität. Diese Resultate weisen auf ein unterschiedliches ATGL-Expressionsprofil in benignen und malignen glattmuskulären Neoplasien. Die ATGL-Expression könnte somit zur Diagnitätsbwertung dieser Tumoren herangezogen werden.

Der Beitrag von Herrn Baumhoer und Mitarbeitern aus Basel (Schweiz) widmet sich der „Wandzusammensetzung“ so genannter einfacher Knochenzysten. Einfache Knochenzysten zeichnen sich durch eine ungewöhnlich homogene, eosinophile, fibrinartige Struktur der Zystenwand aus. Zwölf Fälle einfacher Knochenzysten wurden analysiert (konventionelle enzymhistochemische Färbung und immunhistochemische Färbungen für Pro-Kollagen Typ I und Kollagen Typ III). Die Untersuchung wurde durch eine elektronenmikroskopische Analyse vervollständigt. Interessanterweise zeigte sich, dass das homogene eosinophile Material aus kollagenen Fibrillen und nicht aus Fibrin besteht. Dieses Resultat stellt im Hinblick auf das histologische Erscheinungsbild einen unerwarteten Befund dar.

Frau Ogdie und Mitarbeiter aus Philadelphia/Pennsylvania (USA) berichteten über die Anwendung der so genannten konventionellen binären und multikategorischen „receiver operating characteristic“ (ROC) auf semiquantitative, immunhistochemische Expressionsmuster von Synovialitiden. Die ROC-Analyse stellt die Möglichkeit dar, die Wertigkeit von diagnostischen Tests (insbesondere bei Unterscheidung von zwei Krankheitszuständen) zu beurteilen. Standardisiert wurde die Expression von Ki67, CD68, CD15, Faktor VIII, CD3, CD38 und CD2o. In die Analyse wurden 28 Fälle von rheumatoider Arthritis, 31 Osteoarthrosefälle, 11 Fälle einer septischen chronischen Arthritis, 1o Fälle einer so genannten initialen undifferenzierten Oligoarthritis sowie 22 Fälle von Normalgewebe miteinbezogen. Die multikategorische ROCAnalyse identifizierte Ki67, CD68 (subintimal) und $\mathrm{CD}_{15}$ als jene Marker mit der höchsten Trennschärfe. Diese immunhistochemischen Marker könnten somit für die differenzialdiagnostische Einordnung von chronischen Synovialitiden herangezogen werden.

\section{Korrespondenzadressen}

Prof. Dr. V. Krenn

Zentrum für Histologie, Zytologie und

Molekulare Diagnostik

Max-Planck-Str. 18+20, 54296 Trier

v.krenn@patho-trier.de

PD Dr. K. Hauptmann

Institut für Pathologie,

Charité-Universitätsmedizin Berlin,

Charité Campus Mitte (CCM)

Charitéplatz 1, 10117 Berlin

kathrin.hauptmann@charite.de

Interessenkonflikt. Die korrespondierenden Autoren geben an, dass kein Interessenkonflikt besteht.

\section{Literatur}

1. Krenn V, Morawietz L, Häupl T et al (2002) Grading of chronic synovitis - a histopathological grading system for molecular and diagnostic pathology. Pathol Res Pract 198(5):317-325 